\title{
Plasmodium falciparum parasites overexpressing farnesyl diphosphate synthase/geranylgeranyl diphosphate synthase are more resistant to risedronate
}

\author{
Heloisa B Gabriel1, Mauro F Azevedo², Emília A Kimura¹, Alejandro M Katzin+ \\ 1 Universidade de São Paulo, Instituto de Ciências Biomédicas, Departamento de Parasitologia, São Paulo, SP, Brasil \\ ${ }^{2}$ Universidade Federal de São Paulo, Departamento de Biociências, Santos, SP, Brasil
}

Farnesyl diphosphate synthase/geranylgeranyl diphosphate synthase (FPPS/GGPPS) is a key enzyme in the synthesis of isoprenic chains. Risedronate, a bisphosphonate containing nitrogen (N-BP), is a potent inhibitor of blood stage Plasmodium. Here, we show that $P$. falciparum parasites overexpressing FPPS/GGPPS are more resistant to risedronate, suggesting that this enzyme is an important target, and bisphosphonate analogues can be used as potential antimalarial drugs.

Key words: P. falciparum - FPPS/GGPPS - risedronate - overexpression

It has been estimated that more than 200 million cases of malaria occur annually, resulting in over 400,000 deaths. ${ }^{(1)}$ Among the five Plasmodium species that infect humans, $P$. falciparum results in most cases of morbidity and mortality. Chemotherapy is an important component of control strategies, and the looming resistance against artemisinin and its derivatives, which are the most effective antimalarial drugs, is a serious challenge to the goal of the World Health Organization for the reduction of malaria cases and deaths. ${ }^{(1)}$ Isoprenoid synthesis is a metabolic pathway essential for parasite survival during the erythrocytic cycle and is therefore a potential target for the development of antimalarial drugs. ${ }^{(2-3)}$ An essential step in the synthesis of all isoprenoids is the elongation of the isoprene chain by prenyltransferases, which are classified according to the chain length of the final product and the stereochemistry of the double bond formed by condensations. Among the prenyltransferases, farnesyl diphosphate synthase (FPPS) and geranylgeranyl diphosphate synthase (GGPPS) are the most studied isoprenoidmodifying enzymes in Plasmodium..$^{(4-5)}$

The biosynthesis of farnesyl diphosphate (FPP) and geranylgeranyl diphosphate (GGPP) is catalysed by a single bifunctional enzyme (FPPS/GGPPS) in $P$. falciparum and Toxoplasma gondii. ${ }^{(4-6)}$ The metabolites generated are the main precursors of all secondary products from isoprenoid pathways such as those for vitamin $\mathrm{E},{ }^{(7)}$ vitamin $\mathrm{K},{ }^{(8)}$ carotenoids, ${ }^{(9)}$ ubiquinones, ${ }^{(10)}$ and dolichols. ${ }^{(11)}$

Bisphosphonates are inhibitors of bone resorption applied for the treatment and prevention of osteoporosis. ${ }^{(12)}$ Risedronate, a bisphosphonate containing nitrogen (N-BP),

doi: 10.1590/0074-02760180174

Financial support: FAPESP (no 2014/23417-7, 2017/22452-1).

HBG is the recipient of a post-doctoral fellowship by FAPESP (process $n^{\circ}$ 2015/12054-3) and MFA is funded by FAPESP (process $n^{\circ} 2015 / 19316-3$ and 2016/15298-3).

+ Corresponding author: amkatzin@icb.usp.br

Received 5 April 2018

Accepted 25 July 2018 inhibits $P$. falciparum FPPS/GGPPS enzymatic activity in vitro and has potent activity against blood stages when added to parasites during in vitro cell culture. ${ }^{(4)}$ The inhibitory effect induced by risedronate can be partially reversed by the simultaneous addition of FPP or GGPP during $P$. falciparum culture treatment. ${ }^{(13)}$ A similar inhibitory effect was reported in vivo against murine malaria parasites. In the same study, it was also demonstrated that risedronate was not toxic for animals. ${ }^{(13)}$ In addition, a synergistic effect with nerolidol, a terpene approved by the Food and Drug Administration (FDA) as a food-flavouring agent, demonstrated the potential use of risedronate in combination therapies. ${ }^{(14-15)}$ It has recently been demonstrated that FPPS/GGPPS presents different binding sites for promising new drugs against malaria. $^{(16)}$ In this study, we engineered $P$. falciparum parasites that overexpress FPPS/GGPPS and evaluated its sensitivity to risedronate to investigate whether FPPS/GGPPS is its main target in P. falciparum.

Since FPPS/GGPPS is constitutively expressed in $P$. falciparum, ${ }^{(4)}$ initial attempts aimed to overexpress FPPS/ GGPPS-green fluorescent protein-haemagglutinin (FPPS/ GGPPS-GFP-HA) under the control of the EF1-a promoter. Stably transfected parasites were never recovered, suggesting that overexpression of FPPS/GGPPS-GFP-HA might be toxic. Previous studies have demonstrated toxic effects due to the overexpression of enzymes that use GPP or FPP as substrates, ${ }^{(17-18)}$ caused mainly by the depletion of FPP substrate. In order to keep the overexpression to minimum during the selection of transfected parasites, FPPS/GGPPS was cloned into pRM2-GFP-HA-DD24 (Fig. 1A). ${ }^{(19)}$ The expression as a fusion with HA and the destabilisation domain (DD) would target the fusion protein for degradation, which could only be prevented by the presence of the ligand Shld-1.(20) This strategy was successful, and stable transfection in 3D7 strain parasites generated the transgenic line FPPS/GGPPS-DD-epi.

Given that the transgene in pRM2-derived vectors is under the control of the MSP2 promoter, which is strongly active in schizonts, both RNA and proteins were extracted from transgenic and wild-type (WT) parasites at 
this stage to compare their expressions. FPPS/GGPPS transcript levels were compared by real-time quantitative-polymerase chain reaction (RTq-PCR) (Fig. 1B). As expected, the extra copies of FPPS/GGPPS gene in the transgenic line resulted in significant overexpression, with transcript levels being about 2-fold higher than 3D7 parasites (Fig. 1B). To verify that $\mathrm{DD} /$ Shld-1 regulation played a role, protein was extracted from parasites cultured with $(400 \mathrm{nM})$ or without Shld-1 and analysed by western blotting using antibodies against the HA epitope (Sigma-Aldrich, St. Louis, MO, USA), or PTEX150, ${ }^{(21)}$ which was used as an internal control. FPPS/GGPPSHA-DD24 was only detected in the sample extracted from parasites maintained on Shld-1, demonstrating that the ligand was required to stabilise the protein (Fig. 1C). Due to the lack of a specific antibody, it was not possible to compare FPPS/GGPPS expression between transgenic and WT parasites at the protein level. However, considering the increased transcript levels and that FPPS/ GGPPS-GFP-HA can be easily detected from parasites cultured on Shld-1, this enzyme was likely expressed at higher levels in the transgenic parasites cultured on Shld-1 than in WT parasites.

To investigate whether FPPS/GGPPS expression correlates with resistance to risedronate, 3D7 WT and transgenic parasites at the ring stage were cultured with different concentrations of the drug for $48 \mathrm{~h}$ to determine the $\mathrm{IC}_{50}$ (Fig. 2). Growth was determined by fluorescence method ${ }^{(22)}$ and confirmed by microscopic examination. ${ }^{(23)}$ Shld-1 (400 nM) was added after $24 \mathrm{~h}$ to stabilise the FPPS/GGPPS-HA-DD24 fusion since it was supposed to be expressed in schizonts. The $\mathrm{IC}_{50}$ of $3 \mathrm{D} 7$ and transgenic parasites cultured without Shld-1 was about $20 \mu \mathrm{M}$, suggesting that the transfection process and expression of the selectable marker hDHFR did not affect sensitivity to risedronate. However, in the presence of Shld-1, the $\mathrm{IC}_{50}$ of transgenic parasites increased 1.7 -fold to about $34 \mu \mathrm{M}$, while the sensitivity to risedronate of 3D7 parasites was not affected. Sensitivity to the unrelated drug chloroquine was neither affected by the ligand nor by FPPS/GGPPS overexpression (Supplementary data). Lower Shld-1 concentrations did not affect the $\mathrm{IC}_{50}$, and higher concentrations were not evaluated since they were toxic to the parasites, thereby reducing their growth by about $11 \%$ per reinvasion cycle. ${ }^{(19)}$

Although FPPS/GGPPS-DD-epi parasites have an $\mathrm{IC}_{50}$ significantly higher than that of WT parasites, the resistance phenotype was subtle when compared to the phenotype of other transgenic lines. Overexpression of octaprenyl pyrophosphate synthase/phytoene synthase (OPP/PSY) caused a 5 -fold increase in the $\mathrm{IC}_{50}$ to squalestatin. ${ }^{(24)}$ It was possible that the achieved FPPS/ GGPPS overexpression was not as strong as the OPP/ PSY overexpression, which could be due to incomplete protein stabilisation in the presence of Shld-1 or lower plasmid copy number.

The results presented here corroborates previous findings that FPPS/GGPPS is an important target of risedronate in P. falciparum, suggesting that this compound or a more potent analogue could be developed as an antimalarial drug or be applied in combination therapies in future.
For the plasmid construction, the FPPS/GGPPS coding sequence (PlasmoDB ID: PF3D7_1128400) was synthesised by GenScript, and FPPS/GḠPPS was cloned in pRM2-GFP-HA-DD24, ${ }^{(19)}$ replacing the GFP gene with the Xhol/MluI restriction site to generate pRM2-FPPS/ GGPPS-HA-DD24. Cultures of P. falciparum clone 3D7 were grown as described previously, ${ }^{(25)}$ except that human serum was replaced with Albumax I ( $0.5 \%$; Invitrogen/ Life Technologies, Carlsbad, CA, USA). Parasite multiplication was monitored by microscopic evaluation of Giemsa-stained thin smears. Schizont stages were purified with magnetic columns [magnetically activated cell sorting (MACS) separation columns; CS; Miltenyi Biotec]. ${ }^{(26)}$ Parasites were transfected as previously described ${ }^{(27)}$ using the electroporation conditions established elsewhere. ${ }^{(28)}$

For the cDNA preparation, the RNA was extractedusing TRIzol LS (Invitrogen) following the manufacturer's instructions. Oligonucleotides (GAGTGGGAAAAAGTGGCTTG and CACATCATTCACCGCATTCT) for the detection of FPPS/GGPPS were designed using Primer3

A

pRM2-FPPS/GGPPS-HA-DD24

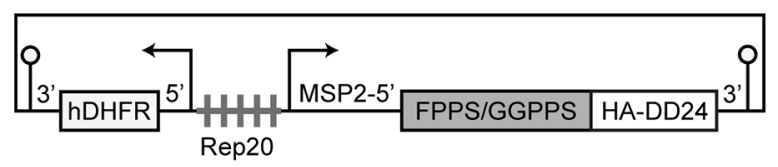

B

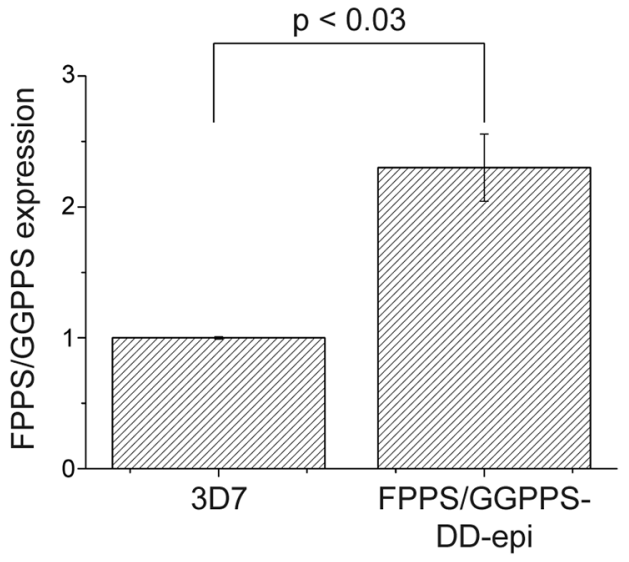

C

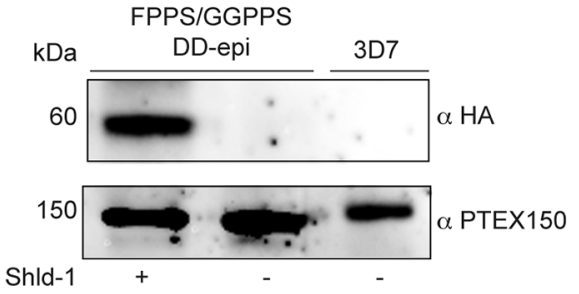

Fig. 1: overexpression of farnesyl diphosphate synthase/geranylgeranyl diphosphate synthase-destabilisation domain-epi (FPPS/GGPPSDD-epi). (A) Schema of plasmid construction used for transfection. (B) Real-time-polymerase chain reaction (RT-PCR) showed overexpression of FPPS/GGPPS-DD-epi compared with wild-type 3D7. (C) Western blot analysis showing the expression of FPPS/GGPPS protein only when Shld-1 was added. Antibody for a constitutively expressed protein (PTEX150) was used as an internal control. Band intensities were measured by ImageJ, density values were evaluated by one-way analysis of variance (ANOVA), and $\mathrm{p}$ values are indicated. 


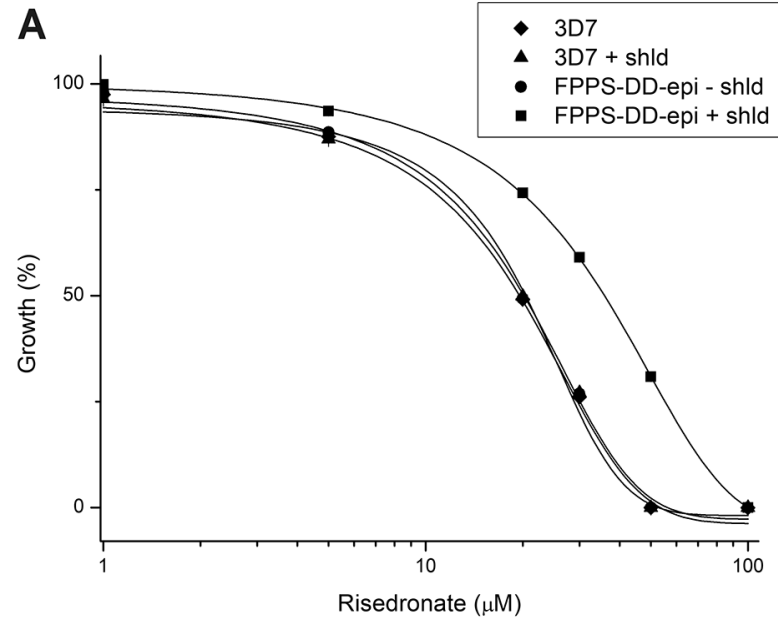

B

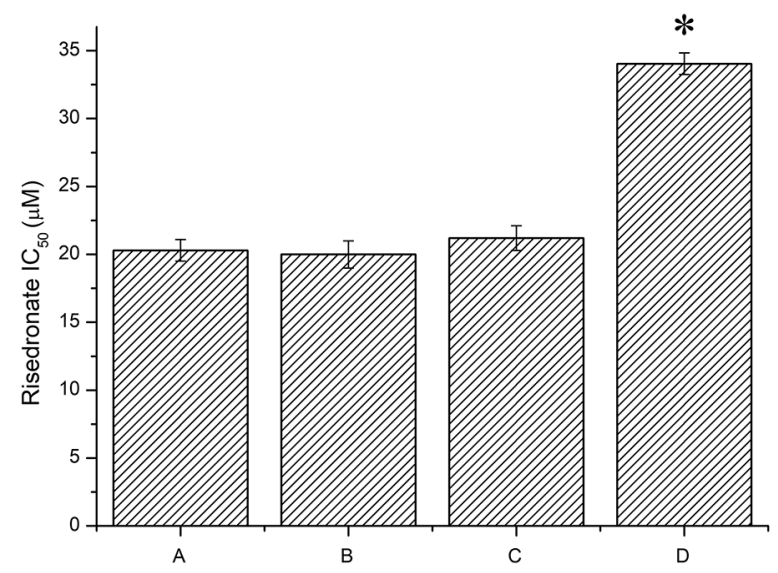

Fig. 2: parasites overexpressing farnesyl diphosphate synthase/geranylgeranyl diphosphate synthase (FPPS/GGPPS) were more resistant to risedronate. Parasites of the wild-type 3D7 strain and the transgenic line FPPS/GGPPS-destabilisation domain-epi (FPPS/GGPPS-DDepi) cultured for two days in the presence of various concentrations of risedronate. (A) Nonlinear regression of parasite growth and risedronate concentrations. (B) $\mathrm{The}_{\mathrm{IC}}$ represents the means with $95 \%$ confidence intervals from three experiments: A-3D7 without Shld-1 $20.3 \pm 0.8 \mu \mathrm{M}, \mathrm{B}-3 \mathrm{D} 7$ with Shld-1 $20 \pm 1 \mu \mathrm{M}, \mathrm{C}-\mathrm{FPPS} /$ GGPPS-DDepi without Shld-1 $21.2 \pm 0.9 \mu \mathrm{M}$, and D-FPPS/GGPPS-DD-epi $34.8 \pm$ $0.8 \mu \mathrm{M}$. Statistical significance was determined by one-way analysis of variance (ANOVA), ${ }^{*} \mathrm{p}<0.02$.

(http://frodo.wi.mit.edu/). The internal control transcript used for calibration throughout the experiments was locus seryl t-RNA transferase (PlasmoDB no. PF3D7 0717700), previously shown as a reliable control. ${ }^{(29)}$ The relative mRNA expression was obtained using the formula $2^{-\triangle C T}$. All experiments were performed in duplicate.

In the inhibition tests, risedronate was dissolved in water, resulting in $25 \mathrm{mM}$ stock solutions. ${ }^{(13)} \mathrm{WT}$ and transgenic parasites at the ring stage were cultured in different concentrations of the drug $(200,20,2,0.2$, and $0.02 \mu \mathrm{M}$ ) for $48 \mathrm{~h}$. Growth was determined by the SYBR Green method $^{(22)}$ and confirmed by microscopic examination. ${ }^{(23)}$. Shld-1 $(400 \mathrm{nM})$ was added after $24 \mathrm{~h}$ to stabilise the FPPS/GGPPS-HA-DD24 fusion. All tests were performed in triplicates from three independent experiments. The $\mathrm{IC}_{50}$ for growth inhibition was calculated by nonlinear regression in GraphPad Prism 5.0 (GraphPad Software, Inc., San Diego, CA, USA).

For western blot analyses, synchronous cultures of 3D7 and transfected parasites with and without Shld-1 at schizont stages were treated with $0.15 \%$ saponin in RPMI media and washed twice with PBS. Proteins were extracted from the parasite pellets after resuspending in buffer containing $0.05 \mathrm{M}$ Tris- $\mathrm{HCl}$ ( $\mathrm{pH} 6.8$ ), $10 \%$ glycerol, $2 \mathrm{mM}$ EDTA, 2\% SDS, $0.05 \%$ bromophenol blue, and $50 \mathrm{mM}$ dithiothreitol ${ }^{(30)}$ for separation by sodium dodecyl sulphate-polyacrylamide gel electrophoresis (SDS-PAGE). The $\alpha$-HA monoclonal antibody (1:500 dilution; Sigma-Aldrich) was used, and $\alpha$-PTEX150 $(1: 1000)^{(21)}$ was used as a control.

\section{ACKNOWLEDGEMENTS}

To S Wendel (Blood bank at the Sírio Libanês Hospital) for providing the erythrocytes.

\section{AUTHORS' CONTRIBUTION}

HLG developed all the experimental work; MFA participated in the work presented in Fig. 1; EAK participated in the work presented in Fig. 2; AMK conceived and supervised the study, analysed data, and wrote the manuscript. All authors read and approved the final version of the manuscript.

\section{REFERENCES}

1. WHO - World Health Organization. World Malaria Report. 2016. Available from: http://who.int/malaria/publications/world-malaria-report-2016/en/.

2. Yeh E, DeRisi JL. Chemical rescue of malaria parasites lacking an apicoplast defines organelle function in blood-stage Plasmodium falciparum. PLoS Biol. 2011; 9(8): e1001138.

3. Wiley JD, Merino EF, Krai PM, McLean KJ, Tripathi AK, VegaRodriguez J, et al. Isoprenoid precursor biosynthesis is the essential metabolic role of the apicoplast during gametocytogenesis in Plasmodium falciparum. Eukaryot Cell. 2015; 14(2): 128-39.

4. Jordao FM, Gabriel HB, Alves JM, Angeli CB, Bifano TD, Breda $A$, et al. Cloning and characterization of bifunctional enzyme farnesyl diphosphate/geranylgeranyl diphosphate synthase from Plasmodium falciparum. Malar J. 2013; 12: 184.

5. Artz JD, Wernimont AK, Dunford JE, Schapira M, Dong A, Zhao $\mathrm{Y}$, et al. Molecular characterization of a novel geranylgeranyl pyrophosphate synthase from Plasmodium parasites. J Biol Chem. 2011; 286(5): 3315-22.

6. Ling Y, Li ZH, Miranda K, Oldfield E, Moreno SN. The farnesyldiphosphate/geranylgeranyl-diphosphate synthase of Toxoplasma gondii is a bifunctional enzyme and a molecular target of bisphosphonates. J Biol Chem. 2007; 282(42): 30804-16.

7. Sussmann RA, Angeli CB, Peres VJ, Kimura EA, Katzin AM. Intraerythrocytic stages of Plasmodium falciparum biosynthesize vitamin E. FEBS Lett. 2011; 585(24): 3985-91.

8. Tonhosolo R, Gabriel HB, Matsumura MY, Cabral FJ, Yamamoto MM, D'Alexandri FL, et al. Intraerythrocytic stages of Plasmodium falciparum biosynthesize menaquinone. FEBS Lett. 2010; 584(23): 4761-8.

9. Tonhosolo R, D'Alexandri FL, de Rosso VV, Gazarini ML, Matsumura MY, Peres VJ, et al. Carotenoid biosynthesis in intraerythrocytic stages of Plasmodium falciparum. J Biol Chem. 2009; 284(15): 9974-85. 
10. de Macedo CS, Uhrig ML, Kimura EA, Katzin AM. Characterization of the isoprenoid chain of coenzyme Q in Plasmodium falciparum. FEMS Microbiol Lett. 2002; 207(1): 13-20.

11. Couto AS, Kimura EA, Peres VJ, Uhrig ML, Katzin AM. Active isoprenoid pathway in the intra-erythrocytic stages of Plasmodium falciparum: presence of dolichols of 11 and 12 isoprene units. Biochem J. 1999; 341(Pt 3): 629-37.

12. Rodan GA. Mechanisms of action of bisphosphonates. Annu Rev Pharmacol Toxicol. 1998; 38: 375-88.

13. Jordao FM, Saito AY, Miguel DC, Peres VJ, Kimura EA, Katzin $\mathrm{AM}$. In vitro and in vivo antiplasmodial activities of risedronate and its interference with protein prenylation in Plasmodium falciparum. Antimicrob Agents Chemother. 2011; 55(5): 2026-31.

14. da Silva MF, Saito AY, Peres VJ, Oliveira AC, Katzin AM. In vitro antimalarial activity of different inhibitors of the plasmodial isoprenoid synthesis pathway. Antimicrob Agents Chemother. 2015; 59(8): 5084-7.

15. Saito AY, Rodriguez AAM, Vega DSM, Sussmann RA, Kimura EA, Katzin AM. Antimalarial activity of the terpene nerolidol. Int J Antimicrob Agents. 2016; 48(6): 641-6.

16. Gisselberg JE, Herrera Z, Orchard LM, Llinas M, Yeh E. Specific inhibition of the bifunctional farnesyl/geranylgeranyl diphosphate synthase in malaria parasites via a new small-molecule binding site. Cell Chem Biol. 2018; 25(2): 185-93.e5.

17. Neudert U, Martinez-Ferez IM, Fraser PD, Sandmann G. Expression of an active phytoene synthase from Erwinia uredovora and biochemical properties of the enzyme. Biochim Biophys Acta. 1998; 1392(1): 51-8

18. Fray RG, Wallace A, Fraser PD, Valero D, Hedden P, Bramley PM, et al. Constitutive expression of a fruit phytoene synthase gene in transgenic tomatoes causes dwarfism by redirecting metabolites from the gibberellin pathway. Plant J. 1995; 8(5): 693-701.

19. de Azevedo MF, Gilson PR, Gabriel HB, Simoes RF, Angrisano F, Baum J, et al. Systematic analysis of FKBP inducible degradation domain tagging strategies for the human malaria parasite Plasmodium falciparum. PLoS One. 2012; 7(7): e40981.
20. Banaszynski LA, Wandless TJ. Conditional control of protein function. Chem Biol. 2006; 13(1): 11-21.

21. de Koning-Ward TF, Gilson PR, Boddey JA, Rug M, Smith BJ, Papenfuss AT, et al. A newly discovered protein export machine in malaria parasites. Nature. 2009; 459(7249): 945-9.

22. Smilkstein M, Sriwilaijaroen N, Kelly JX, Wilairat P, Riscoe M. Simple and inexpensive fluorescence-based technique for highthroughput antimalarial drug screening. Antimicrob Agents Chemother. 2004; 48(5): 1803-6.

23. Desjardins RE, Canfield CJ, Haynes JD, Chulay JD. Quantitative assessment of antimalarial activity in vitro by a semiautomated microdilution technique. Antimicrob Agents Chemother. 1979; 16(6): $710-8$.

24. Gabriel HB, Silva MF, Kimura EA, Wunderlich G, Katzin AM, Azevedo MF. Squalestatin is an inhibitor of carotenoid biosynthesis in P. falciparum. Antimicrob Agents Chemother. 2015; 59(6): 3180-8.

25. Trager W, Jensen JB. Human malaria parasites in continuous culture. Science. 1976; 193(4254): 673-5.

26. Trang DT, Huy NT, Kariu T, Tajima K, Kamei K. One-step concentration of malarial parasite-infected red blood cells and removal of contaminating white blood cells. Malar J. 2004; 3: 7 .

27. Wu Y, Sifri CD, Lei HH, Su XZ, Wellems TE. Transfection of Plasmodium falciparum within human red blood cells. Proc Natl Acad Sci USA. 1995; 92(4): 973-7.

28. Fidock DA, Wellems TE. Transformation with human dihydrofolate reductase renders malaria parasites insensitive to WR99210 but does not affect the intrinsic activity of proguanil. Proc Natl Acad Sci USA. 1997; 94(20): 10931-6.

29. Salanti A, Staalsoe T, Lavstsen T, Jensen AT, Sowa MP, Arnot DE, et al. Selective upregulation of a single distinctly structured var gene in chondroitin sulphate A-adhering Plasmodium falciparum involved in pregnancy-associated malaria. Mol Microbiol. 2003; 49(1): 179-91.

30. Bullen HE, Tonkin CJ, O'Donnell RA, Tham WH, Papenfuss AT, Gould S, et al. A novel family of Apicomplexan glideosomeassociated proteins with an inner membrane-anchoring role. J Biol Chem. 2009; 284(37): 25353-63. 